\title{
Growing genomic research on the African continent: The H3Africa Consortium
}

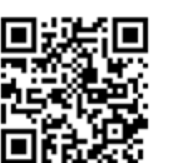

Africa is disproportionately burdened by communicable and non-communicable diseases and their consequences. The 2010 - 2015 life expectancy at birth is 58.2 years for people born in Africa compared with 77.7 years in more developed regions, with a world average of 70.0 years (United Nations, World Mortality Report 2013 $3^{[1]}$ ). The Human Heredity and Health in Africa (H3Africa) Consortium is unique in its objective to promote human genomic research and health in Africa, led and managed by African investigators and their collaborators. ${ }^{[2]}$ In line with the vision articulated in the H3Africa White Paper published in 2011, ${ }^{[3]}$ the H3Africa Consortium aims to build capacity for genomic research in Africa, promote research on both communicable and non-communicable diseases and improve our understanding of the complexities of their coexistence in African populations, generate data and knowledge to promote an understanding of the health and epidemiological transition across the continent, and improve the health of its people. It provides a platform of integrated resources for biomedical research on the continent and is developing an extraordinary wealth of data. In addition it will host biorepositories with samples from over 75000 African participants, many of whom have provided broad consent for sharing their data and samples through a managed access approach, to the benefit of the African and international research community.

The H3Africa initiative was born out of discussions in the African Society of Human Genetics and with key funders to highlight the need to include Africans in global genomic research, and importantly to do so from an African perspective. In its first phase, H3Africa is an initiative funded and supported by the Wellcome Trust (UK) and the National Institutes of Health (USA). The first seven grants were awarded in August 2012 and the Consortium has grown to support 26 research groups from 27 African countries with over 500 investigators, including a pan-African bioinformatics network, H3ABioNet (www.h3abionet.org), and biorepository initiatives in Nigeria, South Africa and Uganda. The Consortium meets every 6 months in venues across the continent, starting in Addis Ababa in August 2012 and moving to Accra, Johannesburg, Cape Town, Dar es Salaam, Zambia, and most recently the USA (Washington DC). The eighth meeting is planned for Dakar in May 2016. A fair distribution of meetings across the breadth and length of Africa has not been easy, and multiple challenges have been encountered including the Ebola epidemic, political instability and social upheaval, reflecting the realities on the continent.

At its inception the H3Africa Consortium developed a series of working groups to meet challenges and to support its members through the process of developing genomic research. This was new territory for many of the groups and in many of the countries. Perhaps the most challenging and unique aspects have been in the ethics arena, where the research envisioned by the Consortium had rarely been conducted on a large scale in Africa. Although some of the concerns are related to inadequate funding and infrastructure, a challenge in all developing countries, the Consortium was sensitive to cultural nuances, perceptions of potential exploitation and the need to promote independence at institutional and national levels, yet aimed to balance this with sensible guidelines that ensure a unified set of principles and operating procedures. Internal debates ranged from how to ensure meaningful community engagement and the challenges of explaining genetics and genomics, to broad informed consent (defined in this context as managed but open access to data and biospecimens), effective engagement with ethics review committees and benefit sharing. To promote fairness in a scenario where the African partners are generally under-resourced and underfunded in terms of infrastructure, institutional and national support and lack of a critical mass of appropriately skilled researchers, some adaptations have been made to the usual business of international funding organisations. ${ }^{[4]}$ These included guidelines stipulating that grants should be managed by the African partners, that projects should include strong cross-national partnerships, and that capacity development should be included as an important component. During the development of policies for data and biospecimen access and sharing, consideration was given to the length of time that the primary research producers would have for exclusive access, also in terms of promoting the use of the samples with effective African partnerships for a period of 3 years before they can be independently accessed through a Data and Biospecimen Access Committee (DBAC). Furthermore, the DBAC will consist of a majority of African members, but will be an independent committee to promote the vision of the H3Africa Consortium.

Information on the projects, the research questions they address and the policies and guidelines are accessible through the H3Africa website (www.h3africa.org). Meetings and activities are documented, including a record of publications associated with H3Africa, as well as opportunities for collaboration that are managed through a co-ordinating centre. The H3Africa projects range from research groups addressing infectious diseases, including genetic determinants of susceptibility to trypanosomiasis and tuberculosis, to studies on the microbiome in cervical cancer and in respiratory diseases. Six projects focus on the rise of non-communicable diseases on the continent, including stroke, diabetes, kidney disease, rheumatic heart disease, obesity and cancer. Their joint resource will include over 50000 African participants with significantly overlapping phenotypic and biomarker data enabling nested studies, meta-analyses and replication cohorts. One of the future goals is the expansion of studies into longitudinal cohorts. Two studies address neurological disorders, including schizophrenia, and one examines drug susceptibility to tuberculosis. There is a collaborative African Genomics Network including investigators from Botswana and Uganda, a bioinformatics network (http://h3abionet.org) that includes 32 research groups across 15 African countries, biorepository research groups, and several projects addressing ethical, legal and social issues related to genomic research in Africa.

The success of H3Africa will be measured by knowledge generation, high-impact publications and the ability of its members to effectively access research funding for genomic research in Africa. Ultimately it is about developing strategies and policies towards reducing the burden of disease among Africans and for global health benefit.

For the H3Africa Consortium to realise its full potential, it requires additional partnerships and the support of African governments to promote sustained funding, maintain valuable resources and ensure that African investigators remain important beneficiaries of the Consortium. The establishment of the Alliance for Accelerating Excellence in Science in Africa by the African Academy of Science is a welcome step towards promoting African-led research aligned to local imperatives ${ }^{[5]}$ African investigators need to prepare themselves 
to be strong players in the global arena of genomic research to ensure that those on the continent can participate fully in the next waves of personalised precision medicine.

\section{Michèle Ramsay}

Director, Sydney Brenner Institute for Molecular Bioscience, University of the Witwatersrand, Johannesburg, South Africa, Professor of Human Genetics, Faculty of Health Sciences, University of the Witwatersand, South African Research Chair in Genomics and Bioinformatics of African Populations, and member of the Steering Committee of the H3Africa Consortium
Corresponding author: M Ramsay (michele.ramsay@wits.ac.za)

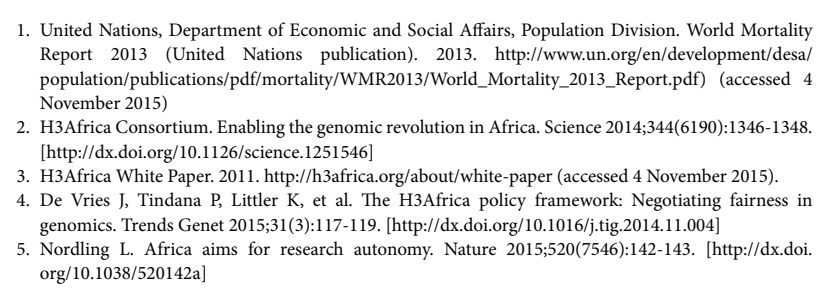

S Afr Med J 2015;105(12):1016-1017. DOI:10.7196/SAMJ.2015.v105i12.10281

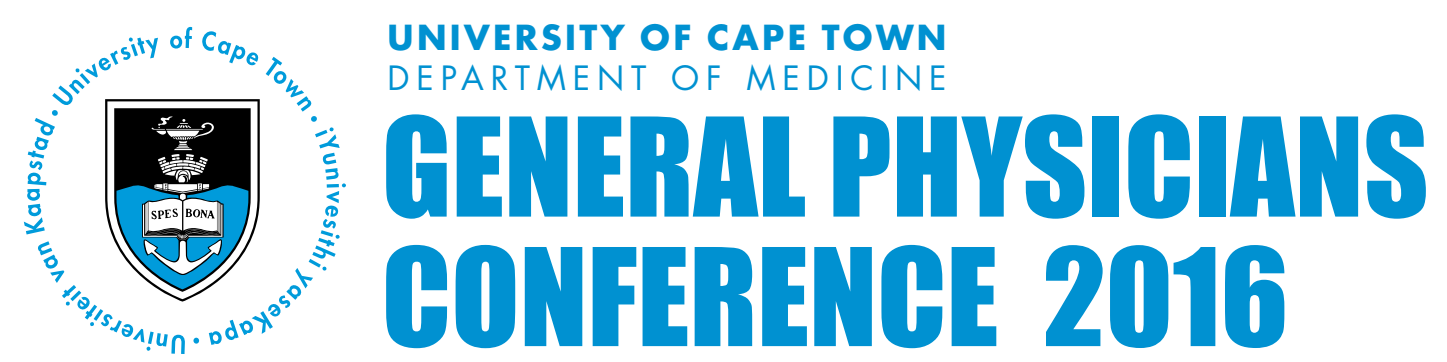

THURSDAY 18 - SUNDAY 21 FEBRUARY-2016 iti CAPE TOWN INTERNATIONAL CONVENUION GENTRE

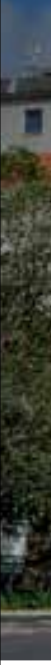

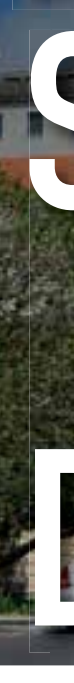

UNIVERSITY OF CAPE TOWN

\section{BONFERENGE 2016}

AN INTERACTIVE

APPROACH TO COMMON MEDICAL DISORDERS AND EMERGENCIES

The conference will bring together a panel of expert speakers from Academic Institutions in Southern Africa.

\section{INTERACTIVE}

SESSIONS,QUIZZES AND UPDATES

The topics will be relevant to specialist physicians and trainees, in the private and public sectors, and colleagues beyond our borders.

\section{'MEET THE EXPERTS'} WORKSHOPS

18 February, Groote Schuur Hospital Topics will include the fields of: Allergology and Clinical Immunology, Cardiology, Dermatology, Diabetes and Endocrinology, Gastroenterology, Geriatrics, Haematology, Hepatology Infectious Diseases and HIV Medicine, Lipidology, Medical Ethics, Nephrology, Neurology, Pharmacotherapy, Pulmonology, and Rheumatology. 